\title{
Numerical Model for Fluid- Structure Interaction by the Coupled SPH and the FEM Method
}

\author{
Alen Harapin, Jure Radnić, Marina Sunara Kusić \\ University of Split, Faculty of Civil Engineering, Architecture and Geodesy, Matice hrvatske 15, 21000 Split, CROATIA \\ e-mail: alen.harapin@gradst.hr
}

\section{SUMMARY}

The paper presents the numerical model developed for the simulation of the fluid-structure interaction problem. The model is based on the so called "partition scheme", in which the Smoothed Particle Hydrodynamics (SPH) method is used for the fluid and the standard Finite Element Method (FEM), based on shell elements, is used for the structure. Then, the two solvers are coupled to obtain the behaviour of the coupled fluid-structure system. The effects of large displacements and small strains are taken into account in the model for shells. The elasto-plastic material model for the structure (shell), which includes some important nonlinear effects like yielding in compression and tension, is briefly discussed. Some of the model's possibilities are illustrated in a practical example of a rectangular medium sized fluid tank with rigid and deformable walls under several ground excitations.

KEY WORDS: liquid tanks; numerical model; coupled problem; smoothed particle hydrodynamics; shell structures.

\section{INTRODUCTION}

In engineering practice structures which are in direct contact with fluid are very common. Those are, for example: dams, water tanks, off shore structures, pipelines, water towers, etc. Numerical simulation of these structures is a very complex task because it involves the simulation of two completely different media: the deformable structure and the fluid. This complexity is particularly emphasized when the system is under dynamic (seismic) conditions, and it is commonly referred to as a fluid-structure interaction (FSI), a coupled or a multi-field problem [1,2].

Investigation of FSI problem probably began with Friedrich Bessel and the so-called concept of "hydrodynamic or added mass" in 1828. He investigated the movement of a pendulum in a fluid and realized that the pendulum had a longer period of oscillation than in a vacuum. He correctly concluded that the surrounding fluid in some way increases the effective mass of the system $[3,4]$. 
In 1843, Sir George Gabriel Stokes conducted a study of a uniform acceleration of a cylinder of infinite length that was moving in an infinite fluid medium. He concluded that the effective mass of the cylinder increased due to the influence of the surrounding fluid, just proportionally to the weight of water it displaced. This discovery is considered to be the beginning of the problem of FSI [4].

In the 1960s, some nuclear power plant designers found that the hydrodynamic mass of a structure in a restricted fluid medium was considerably higher than for a structure in an unrestricted (infinite) fluid medium, which confirmed Stokes' assumptions. The investigation of interactions in pressure tanks and pipelines started in that decade. Fritz and Kiss conducted the first research of the vibration of a cantilever cylinder immersed in a fluid, which is considered to be pioneer research of the FSI problem for power plants [4].

Two main approaches exist for the simulation of fluid-structure interaction problems [5]:

- The monolithic approach: the equations governing the fluid's pressures and the displacements of the structure are solved simultaneously, with a single solver;

- The partitioned approach: the equations governing the fluid's pressures and the displacements of the structure are solved separately, with two distinct solvers.

The monolithic approach requires the development of a specific model for a particular physical problem. The main advantage of the monolithic approach is that no coupling iterations within the time step are required. The partitioned approach allows solving the equation for the fluid and the equation for the structure by different models, possibly more efficient techniques, developed specifically for solving the fluid or the structure problem $[5,6]$. Then, these models are coupled for the solution of the FSI problem.

Thus the partitioned approach has various advantages: (i) the resulting model is very modular, (ii) it's easy to make any modifications, (iii) every modification in one field improves the whole model, (iv) the experts specialized in particular fields can participate.

In the last 30 years, the problem of interaction between the fluid and the structure has been treated by many authors from many scientific and engineering areas: astrophysics, shipbuilding, mechanical engineering and civil engineering. Strong incentives were certainly given by the development of meshfree methods, which can very effectively describe the motion of the fluid. One of the oldest and probably the most famous is the so-called SPH method, originally developed by Gingold and Monaghan [19] and Lucy [18], to describe the astrophysics problem. A number of other methods have been suggested since then, such as: Diffuse element method (1992.); Dissipative particle dynamics (1992.); Element free Galerkin method (1994.); Finite pointset method (1998.); Finite mass method (2000.); Smoothed point interpolation method (2005.); Meshfree local radial point interpolation method; Local radial basis function collocation method; Viscous vortex domains method; Discrete least squares meshless method (2006.); Optimal Transportation Meshfree method (2010.); Repeated replacement method (2012.); and many others [3].

With these methods, it is possible to describe problems in which significant mesh distortions occur during the simulation, which is particularly suitable for liquid flow problems. The disadvantage of all these methods is the need for powerful computers and the extremely long running time of the calculation.

This paper presents a coupled particle and finite element method for the analysis of fluid structure interaction problems involving large displacements in the structure. The SPH method $[3,7,8]$ is used to simulate the fluid and the finite shell element with six degrees of 
freedom per node $[9,10,11]$, which has been freed of the negative influences of shear and membrane locking, is used to simulate the shell structures $[9,10]$.

The SPH method uses the Lagrangian description to model fluid particles. In fluid dynamics the Lagrangian description of the flow field is a way of looking at fluid motion where the observer follows an individual fluid particle as it moves through space and time. Plotting the position of an individual particle through time gives the path line of the particle. This removes difficulties in the treatment of the convective terms. The movement of each particle is computed from the interaction forces between the particles. Furthermore, since the SPH is a particle method, there is no need for numerical grids or meshes. Incompressibility is satisfied implicitly [3]. The SPH method guarantees mass conservation and no numerical diffusion of the free surface [3].

In spite of the very hard work of many scientists, a comprehensive study of such problems remains a challenge due to their multidisciplinary nature $[12,13,14]$. For most FSI problems analytical solutions of the model equations are impossible to solve and laboratory experiments are limited in scope. With advances in hardware and software technology, numerical simulations and numerical methods become a powerful tool for these problems.

\section{NUMERICAL MODEL FOR THE FLUID}

\subsection{FLUID AND NAVIER-STOKES EQUATIONS}

A fluid is a substance (either a liquid or a gas) that continuously deforms under the external loads and redistribute itself in space. Thus, particle methods as the SPH (and many others) are ideal for simulating free surface fluid flow, because it is very easy to follow the path of each particle without remeshing, which is necessary in mesh-based methods [3]. Fluid flow may be classified as either inviscid or viscous. Inviscid flows are frictionless flows characterized by zero viscosity. No real flows are inviscid, but there are numerous fluids and flow situations in which viscous effects can be neglected, for example: water flow far from solid surfaces [15]. Inviscid flows may be further classified as either compressible or incompressible, depending on whether density variations are large or relatively negligible.

The governing equations for the simulation of fluid behaviour are the Navier-Stokes equations, which are a set of equations that describe the motion of fluid, over time. The basic Lagrangian formulation for an incompressible fluid is given by $[3,16,17,18]$ :

$$
\rho \frac{d v_{i}}{d t}=\rho R_{i}-\nabla p+\mu \nabla^{2} v_{i}
$$

In the above equation $v_{i}$ represents the vector of velocity, $\rho$ is the mass-density, $p$ is the pressure, $\mu$ is the dynamic viscosity of the fluid, and $R_{i}$ is the sum of mass-volume forces acting on the fluid. The Navier-Stokes equations are a base point for all fluid movement simulations. For complex situations, involving cavitation, turbulence, aerodynamics, hydrodynamics etc., the approximated solution of the Navier-Stokes equations can only be found by using a computer and with additional simplifications.

\subsection{SMOOTHED PARTICLE HYDRODYNAMICS (SPH)}

The SPH is a meshfree particle method (MPM) which employs a set of a finite number of discrete particles to describe the movement of the system and represent the state of the 
system. Each particle is usually associated with some physical characteristic which is related to a specific problem (mass, energy, position, density, pressure...) and represents a part of the continuum domain. The domain can range from very large astronomical scale, where particles represent stars and planets, through macro scale to micro scale, where particles represent molecules and atoms $[3,18,21]$.

Depending on the used mathematical model, the meshfree particle method can be deterministic or probabilistic. The deterministic MPMs use the governing system equation of physical law directly. In the deterministic MPMs once the initial and boundary conditions are given, the particle evolution in the later time stages can be precisely determined based on the physical laws that define the problem. The probabilistic MPMs also use the governing system equation of physical law but their approach is based on statistical principles. The SPH method has mixed features. It was initially developed as a probabilistic method and was later modified and applied as a deterministic meshfree particle method [18].

The advantages of the SPH method (and all other MPMs) over conventional grid-based methods can be summarized as [3, 22]:

- In the SPH the problem domain is described with a cloud of particles which don't have a fixed connectivity. Thus, the treatment of large movements and large deformations is relatively much easier;

- After the movement, the refinement of the particles is much easier than the refinement of the finite element mesh;

- Discretization of complex geometry is relatively simpler, and only the initial discretization is required;

- It is easy to obtain the features of the entire physical system tracing the motion of the particles.

Also, the SPH method has some disadvantages [3, 22]:

- Boundary condition implementation is a hard task and fluid particles penetration into boundaries must be avoided;

- The interpolation method used in SPH is very simple and it will be strongly affected by particle disorder;

- The method is typically slower computationally when compared to other modern gridbased methods, as the time step is based on the speed of sound in the fluid, although new research has been developed during the last few years to overcome these limitations [33, 34].

The base principle of the SPH method is the approximation of any function $A(\vec{r})$ with a unique, uniform, smooth and compact kernel function $W\left(\vec{r}-\vec{r}^{\prime}, h\right)$ that is defined all over the space $\Omega$, with the smoothing length $h$ :

$$
A(\vec{r})=\int_{\Omega} A\left(\vec{r}^{\prime}\right) W\left(\vec{r}-\vec{r}^{\prime}, h\right) d \vec{r}
$$

where $\vec{r}$ is any point in $\Omega, \vec{r}^{\prime}$ is a neighbouring point and $h$ is the smoothing length or core radius. Smoothing length is a scaling factor that controls the smoothness or roughness of the kernel. The obtained results significantly depend on the choice of the kernel function (Figure 
1). This integral representation in discrete notation leads to the approximation of the function $A(\vec{r})$ at a particle of interest:

$$
A(\vec{r})=\sum_{b} m_{b} \frac{A_{b}}{\rho_{b}} W\left(\vec{r}_{a}-\vec{r}_{b}, h\right)
$$

where the summation is over all of the neighbouring particles $b$ within the region of the kernel function. The mass and the density are denoted by $m_{b}$ and $\rho_{b}$, respectively.

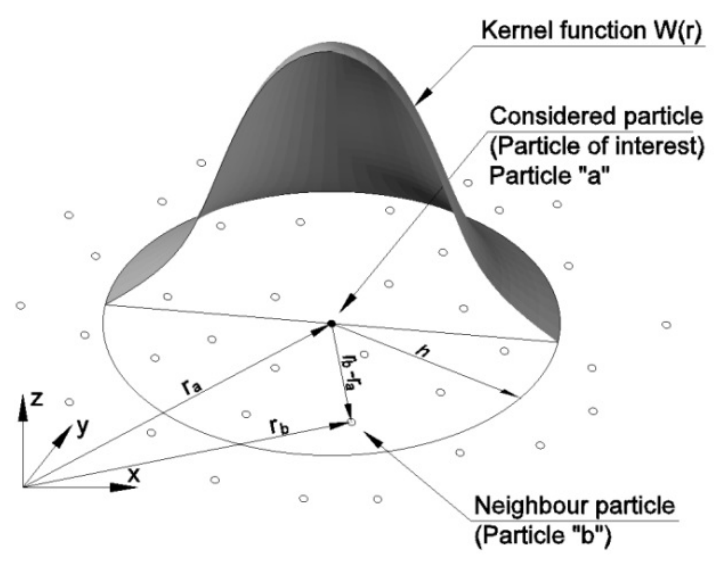

Fig. 1 Kernel function

The kernel function should satisfy several conditions: positivity, compact support and normalization. Also, it has to be monotonically decreasing with increasing distance from the particle and behave like a delta function as the smoothing length tends to zero. One of the most often used kernel function is the cubic spline $[3,22]$, which is also used in this work.

One of the governing equations of fluid dynamics is the momentum conservation equation that in SPH form is:

$$
\frac{d \vec{v}_{a}}{d t}=\sum_{b} m_{b}\left(\frac{P_{b}}{\rho_{b}^{2}}+\frac{P_{a}}{\rho_{a}^{2}}+\Pi_{a b}\right) \vec{\nabla} W_{a b}+\vec{g}
$$

where $g$ is the gravitational acceleration, $P_{a}$ and $P_{b}$ are the pressures corresponding to particles $a$ and $b$ and $\rho_{a}$ and $\rho_{b}$ are the densities corresponding to particles $a$ and $b$ and $\Pi_{a b}$ is the viscosity term. Different approaches, based on various existing formulations of the viscosity terms, can be considered in the SPH method to describe the momentum equation. Two main options for viscosity, which are implemented in the model, are artificial viscosity and laminar viscosity [3,21,22]. In this study, the fluid is considered as inviscid.

The SPH method assumes that the masses and the mass-densities for all particles are known before the method starts. Particle mass is a user defined constant, but mass-density is a continuous field of the fluid, which must be computed [3,22].

Changes in fluid density are calculated according to the continuity equation:

$$
\frac{d \rho_{a}}{d t}=\sum_{b} m_{b} \vec{v}_{a b} \vec{\nabla} W_{a b}
$$


In SPH formalism the fluid is treated as weakly compressible, so fluid pressure is a function of density variations through an equation of state. In this work, Tait's equation is used for the equation of state:

$$
P=\frac{c_{0}^{2} \rho_{0}}{\gamma}\left[\left(\frac{\rho}{\rho_{0}}\right)^{7}-1\right]
$$

where $\gamma=7, B=c_{0}^{2} \rho_{0} / \gamma, \rho_{0}$ is the reference density ( $\rho_{0}=1000 \mathrm{kgm}^{-3}$ for water) and $c_{0}$ is the speed of sound at reference density. Pressure is directly coupled to density through the equation of state. This approach has the disadvantage that the pressure field of the particles has large oscillations. One of the ways to overcome this problem is density correction, which means re-assigning a density to each particle in every $\mathrm{N}$ time step. In this work, the Moving Least Squares (MLS) approach $[3,7,22]$ is applied. New density is defined by:

$$
\rho_{a}^{\text {new }}=\sum_{b} \rho_{b} W_{a b}^{M L S} \frac{m_{b}}{\rho_{b}}=\sum_{b} m_{b} W_{a b}^{M L S}
$$

The kernel is corrected as follows:

$$
W_{a b}^{M L S}=W_{b}^{M L S}\left(\vec{r}_{a}\right)=\beta\left(\vec{r}_{a}\right) \cdot\left(\vec{r}_{a}-\vec{r}_{b}\right) W_{a b}
$$

where the correction vector $\beta$ is defined by:

$$
\beta\left(\vec{r}_{a}\right)=A^{-1}\left[\begin{array}{l}
1 \\
0 \\
0 \\
0
\end{array}\right] \text {, where } A=\sum_{b} W_{b}\left(\vec{r}_{a}\right) \tilde{A} V_{b}
$$

with matrix $\tilde{A}$ :

$$
\tilde{A}=\left[\begin{array}{cccc}
1 & \left(x_{a}-x_{b}\right) & \left(y_{a}-y_{b}\right) & \left(z_{a}-z_{b}\right) \\
\left(x_{a}-x_{b}\right) & \left(x_{a}-x_{b}\right)^{2} & \left(x_{a}-x_{b}\right)\left(y_{a}-y_{b}\right) & \left(x_{a}-x_{b}\right)\left(z_{a}-z_{b}\right) \\
\left(y_{a}-y_{b}\right) & \left(x_{a}-x_{b}\right)\left(y_{a}-y_{b}\right) & \left(y_{a}-y_{b}\right)^{2} & \left(y_{a}-y_{b}\right)\left(z_{a}-z_{b}\right) \\
\left(z_{a}-z_{b}\right) & \left(x_{a}-x_{b}\right)\left(z_{a}-z_{b}\right) & \left(y_{a}-y_{b}\right)\left(z_{a}-z_{b}\right) & \left(z_{a}-z_{b}\right)^{2}
\end{array}\right]
$$

In this work, density correction algorithm is applied every $\mathrm{n}$ time steps [8], where $n$ is a natural number, often 20-30. This correction significantly improves pressure distributions, especially close to the solid boundaries.

Furthermore, the fluid has to the satisfy energy conservation equation:

$$
\frac{d e_{a}}{d t}=\frac{1}{2} \sum_{b} m_{b}\left(\frac{P_{b}}{\rho_{b}^{2}}+\frac{P_{a}}{\rho_{a}^{2}}+\Psi_{a b}\right) \vec{v}_{a b} \vec{\nabla} W_{a b}
$$

where $\Psi_{a b}$ is the viscosity term.

The standard predictor-corrector scheme is adopted as the solver algorithm [3,22]. The timestep control involves the CFL condition, the force terms and the viscous diffusion term [11]. A variable time step is calculated according to [12]: 


$$
\Delta t=0.3 \min \left(\Delta t_{f}, \Delta t_{c v}\right) ; \Delta t_{f}=\min \left(\sqrt{h /\left|f_{a}\right|}\right) ; \Delta t_{c v}=\min \frac{h}{c_{s}+\max \left|\frac{h \vec{v}_{a b} \vec{r}_{a b}}{\vec{r}_{a b}^{2}}\right|}
$$

Here $\Delta t_{f}$ is based on the force per unit mass $\left|f_{a}\right|$, and $\Delta t_{c v}$ combines the Courant and the viscous time step controls.

The boundary of the fluid domain, which represents tank geometry in this work, is described by a set of so called dynamic boundary particles $[3,8]$. These boundary particles are put in several rows (often two) and forced to satisfy the same equations as fluid particles. They are connected to the structure and move according to the displacements of the structure, which will be explain later.

\section{NUMERICAL MODEL FOR THE STRUCTURE}

\subsection{INTRODUCTION}

Shell structures are thin surface structures whose thickness is relatively small in comparison with the other two dimensions. Examples of shell structures are widespread in nature: seashells, snail-houses, turtle shells and so on. So it's logical that the presence of shells is also common in the engineering practice, especially because shells are economically efficient structures.

In engineering practice the term shell refers only to a curved thin structure while other flat structures are called plates which are only a simplified case of shells.

In this work, the so called degenerated shell elements with 8 (Serendipity) or 9 (Lagrangian) nodes, based on the Mindlin-Reissner theory $[9,10,24]$ are used, with 6 degrees of freedom in each node (Figure 2).The behaviour of these elements in shear-weak shell problems is usually very good and it is only in shear-stiff shells that the problem of "shear locking" arises. This problem was solved by selective integration $[9,10]$. The problem of "membrane locking" was solved in a similar manner.

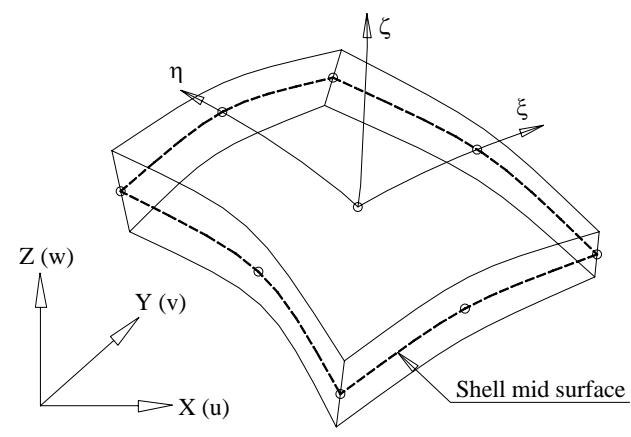

Fig. 2 Shell element

\subsection{NUMERICAL PROCEDURE}

For the dynamic equilibrium of a solid body in motion the Principle of virtual work can be used to write the equations independent of material behaviour: 


$$
\int_{\Omega}(\delta \underline{\varepsilon})^{T} \underline{\sigma} d \Omega-\int_{\Omega}(\delta \underline{u})^{T}\left(b-\rho_{s} \underline{\ddot{u}}-\mu^{\prime} \underline{\dot{u}}\right) d \Omega-\int_{\Gamma_{t}}(\delta \underline{u})^{T} t d \Gamma=0
$$

where $\delta \underline{u}$ is the vector of virtual displacements, $\delta \underline{\varepsilon}$ is the vector of associated virtual strains, $b$ is the vector of applied body forces, $t$ is the vector of surface tractions, $\underline{\sigma}$ is the vector of stresses, $\rho_{s}$ is the mass density, $\mu^{\prime}$ is the damping parameter. The domain of interest $\Omega$ has the boundary $\Gamma_{t}$ on which boundary tractions t are specified.

In dynamic analysis, the finite element method can be applied in both analyses: for space and for time. However, it is general practice to use finite elements for space and finite differences for time $[9,10,24,25]$. This approach is also adopted in this work. Here, the displacement formulation is used because of its simplicity, generality and good numerical properties. For the representation of the finite element, the displacements and the strains and also their virtual counterparts are given by the following relationships:

$$
\begin{array}{lll}
\underline{u}=\mathbf{N u} & \partial \underline{u}=\mathbf{N} \partial \mathbf{u} \\
\underline{\varepsilon}=\mathbf{B u} & \partial \underline{\varepsilon}=\mathbf{B} \partial \mathbf{u} \\
\underline{\sigma}=\mathbf{D} \underline{\varepsilon}=\mathbf{D B u} \mathbf{u} & \partial \underline{\sigma}=\mathbf{D} \partial \underline{\varepsilon}=\mathbf{D B} \partial \mathbf{u}
\end{array}
$$

where $u$ is the vector of nodal displacements, $\partial \mathbf{u}$ is the vector of virtual nodal variables, $N$ is the matrix of global shape functions, $B$ is the strain-displacement matrix and $D$ is the constitutive matrix $[9,10]$.

If (14) are substituted into (13), and if we note that the resulting equation is true for any set of virtual displacements, then the following equation can be obtained in a well-known matrix form:

$$
\mathbf{M}_{s} \ddot{u}_{i}+\mathbf{C}_{s} \dot{u}_{i}+\mathbf{R}_{s}\left(u_{i}\right)=\mathbf{f}_{s}+\mathbf{M}_{s} \ddot{d}
$$

where:

$$
\begin{aligned}
& \left(\mathbf{M}_{s}\right)_{k j}=\int_{\Omega_{s}} \mathbf{N}_{s k}^{T} \rho_{s} \mathbf{N}_{s j} d \Omega \\
& \left(\mathbf{C}_{s}\right)_{k j}=\int_{\Omega_{s}} \mathbf{N}_{s k}^{T} \mu^{\prime} \mathbf{N}_{s j} d \Omega \\
& \mathbf{R}_{s}\left(u_{i}\right)=\int_{\Omega_{s}} \mathbf{B}^{T}\left(u_{i}\right) \sigma_{i} d \Omega \\
& \left(\mathbf{f}_{s}\right)_{i}=\int_{\Omega_{s}} \mathbf{N}_{s k}^{T} \mathbf{b}_{i} d \Omega+\int_{\Gamma_{t}} \mathbf{N}_{s k}^{T} \mathbf{t}_{i} d \Gamma
\end{aligned}
$$

where $N$ is the matrix of global shape functions $[9,10,24], \boldsymbol{M}_{s}, \boldsymbol{C}_{s}, \boldsymbol{R}_{s}\left(u_{i}\right)$ are the well-known matrices of mass, damping and stiffness, respectively, and $\ddot{d}$ is the ground acceleration caused by an earthquake.

The strain-deformation relationship is assumed non-linear:

$$
\varepsilon=\mathbf{B} u \quad ; \quad \mathbf{B}=\mathbf{B}(u)
$$

which represents the so called geometrical nonlinearity. In fact, the matrix $B$ is not linear and dependents on system displacements. 


\subsection{SIMULATION OF MATERIAL BEHAVIOUR}

All real materials have nonlinear behaviour, but in many cases the material can be consider linear. Many types of material models were developed to represent the variety of behaviours such as linear elastic, nonlinear elastic, elasto-plastic, visco-elastic, visco-plastic, creep, cracking or fracture etc. Different hardening laws such as isotropic and kinematic hardening also developed in the plastic and visco-plastic models. Apart from the linear elastic models, all of these representations are, in some sense, nonlinear.

In physics and materials science, term "plasticity" describes the deformation of a (solid) material undergoing non-reversible changes of shape in response to applied forces. In this work, a material model called "Anisotropic yielding" is implemented. This material model can describe very well the behaviour of some real materials such as metals, especially steel, some kinds of artificial materials (PE, PEHD) and so on.

The theory of elasto-plastic behaviour is governed by three conditions: the initial yield condition, the flow rule and the hardening rule. In this work, a generalization of the HuberMises law $[9,26]$ was adopted, with the general form:

$$
F(\sigma, k)=f(\sigma)-Y(k)
$$

where $f(\sigma)$ is the certain function of deviatoric stress invariants and $Y(k)$ is the yield level which is commonly a function of the hardening parameter $k$. If we assume that the traverse normal stress $\sigma_{3}=0$, which is common in shells, the $f(\sigma)$ can be written in the following form [9]:

$$
f(\sigma)=\left(\alpha_{1} \sigma_{1}^{2}+\alpha_{2} \sigma_{1}^{2}+2 \alpha_{12} \sigma_{1} \sigma_{2}+\alpha_{3} \tau_{12}^{2}+\alpha_{4} \tau_{13}^{2}+\alpha_{5} \tau_{23}^{2}\right)^{1 / 2}
$$

In eq. (19) $\alpha_{1}, \alpha_{2}, \alpha_{12}, \alpha_{13}, \alpha_{23}$, are isotropic parameters, which can be determined experimentally by six independent yield tests. The subscripts 1,2, 3 refer to the direction of the three principal axis of anisotropy. These isotropic parameters can be, for example, found in [9, $27,28,29]$.

The total strain increment, $\mathrm{d} \varepsilon$ is the sum of the elastic and the plastic components, thus:

$$
d \varepsilon=d \varepsilon_{e}+d \varepsilon_{p}
$$

where subscripts $e$ and $p$ denote the elastic and the plastic component, respectively. The plastic strain increment can be written in the following form [9]:

$$
d \varepsilon_{p}=d \lambda \frac{\partial Q}{\partial \sigma}
$$

where $d \lambda$ is the non-negative scalar. For the associated flow the potential $Q$ is taken identical to the yield function $f(\sigma)$, expression (19). The differential form (18) can be written as:

$$
\mathbf{a}^{T} d \sigma-A d \lambda=0
$$

where $a$ represents the flow vector and $A$ is the hardening parameter. The exact expression to calculate $a$ and $A$ can be found in $[9,27]$. For linearly hardening materials, such as steel, the hardening parameter is constant. If the material is ideally plastic, the hardening parameter is equal to zero.

Finally, the elasto-plastic incremental stress-strain relationship can be written in the following form: 


$$
d \sigma=\mathbf{D}_{e p} d \varepsilon_{p}
$$

where $D_{e p}$ represents the matrix of elasto-plastic coefficients, and can be written in following form:

$$
\mathbf{D}_{e p}=\mathbf{D}-\frac{\mathbf{D a a}^{T} \mathbf{D}}{A+\mathbf{a}^{T} \mathbf{D a}}
$$

The matrix $D$ represents the matrix of elastic coefficients and it is a function of the elastic (Young's) modulus and the Poisson's coefficients. The exact expression for this matrix can be found in $[9,27]$.

\subsection{SIMULATION OF LARGE DISPLACEMENTS}

The geometric nonlinearity of the structure is the result of: the effects of changes in its geometry (due to the displacement field) and the non-linear connection between the strains and the displacement.

The so-called linear geometry model does not take into account the impact of changes in the geometry of the structure. Specifically, in this model, the equilibrium equations are satisfied on the undeformed (initial) system. Such an approach is acceptable for a rigid structure in which the system deformed a little (Small displacements problem). When the construction has large displacements (Large displacements problem), the equilibrium equations need to be satisfied on the deformed system, because the application of the linear model geometry gives unsatisfactory results.

The application of the small displacements model in the calculation of "slender" structures may lead to wrong conclusions in their capacity and safety. Sometimes they are less safe (if the longitudinal compressive forces prevail), and sometimes they are on the side of greater safety (if the longitudinal tensile forces prevail). In some cases the linear geometry model is unusable and using the nonlinear model is a necessity. The whole procedure of the simulation of large displacements is described in detail in $[11,26]$.

\section{SOLUTION CONCEPT FOR THE DYNAMIC FLUID-STRUCTURE INTERACTION PROBLEM}

As mentioned before, in the partitioned approach the two developed models/softwares are independent. In this work, the previously developed software based on the SPH [22] is used for analysis of fluid and the previously developed model based on the FEM $[10,11]$ is used for analysis of structure. Both programs are written in FORTRAN on PC platform. Those programs are bind together and two softwares communicate, and exchange information.

During the deformation of the structure, the computational domain of the fluid is constantly changing. This also changes the forces of the fluid on the structure in relation to the initial state, which creates a repeating causal-consequent process (Figure 3). 

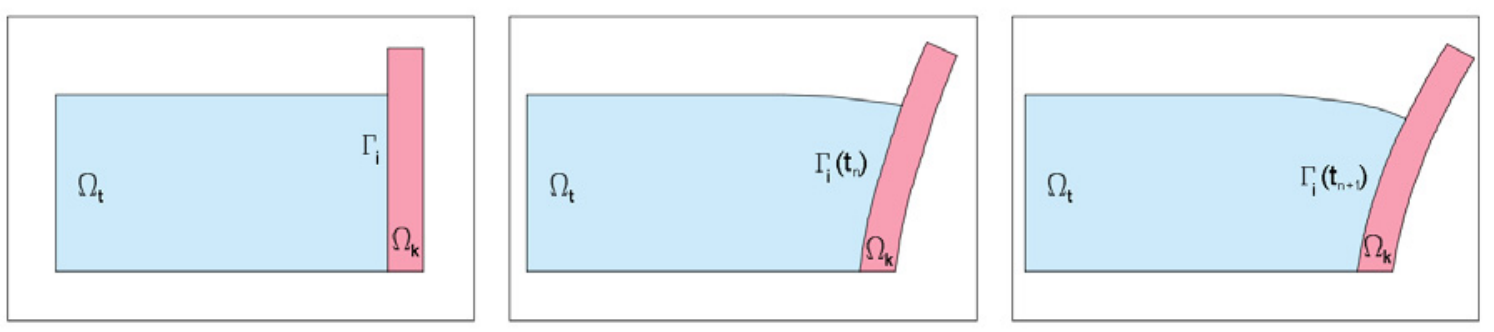

Fig. 3 Fluid-structure interaction

The first step in the numerical analysis is to determine the fluid pressures on the structure for the initial state (steady state). In some cases those are the hydrostatic pressures and in some cases the initial pressures are equal to zero. The next step is solving equation (15), with one additional member: $\mathrm{f}_{\mathrm{fs}}$, which represents the fluid forces on the structure.

$$
\mathbf{M}_{s} \ddot{u}_{i}+\mathbf{C}_{s} \dot{u}_{i}+\mathbf{R}_{s}\left(u_{i}\right)=\mathbf{f}_{s}+\mathbf{M}_{s} \ddot{d}+\mathbf{f}_{f s}
$$

where $f_{s}$ is external force vector. The vector of fluid forces $f_{f s}$, can be calculated from:

$$
\mathbf{f}_{f s}=-\mathbf{Q} \mathbf{P}
$$

where $P$ represents the fluid pressures on the structure and $Q$ is interaction matrix, which defines the interface between fluid and structure. So, the vector of fluid forces $f_{f s}$ is the result of the pressures $P$ in the fluid and is calculated based on the values of the pressures in the fluid particles located in the immediate vicinity of the considered structure node of the interaction surface. All of the fluid particles found within the spatial distance of $2 h$ of the observed considered structure node (Figure 4) contribute in the calculation. In this paper, the correction of pressure on the borders of the domain was adopted according to expression:

$$
P_{a}=\frac{\sum_{b} \frac{P_{b}}{\vec{r}_{b}-\vec{r}_{a}}}{\sum_{b} \frac{1}{\vec{r}_{b}-\vec{r}_{a}}}
$$

where $a$ represents the boundary particle of interest, and $b$ represents the neighbouring fluid particles (within the compact domain $\Omega$ ) (see Figure 1). Averaging the fluid pressures at the boundaries according to (27), the unphysical oscillations of pressures are significantly reduced.

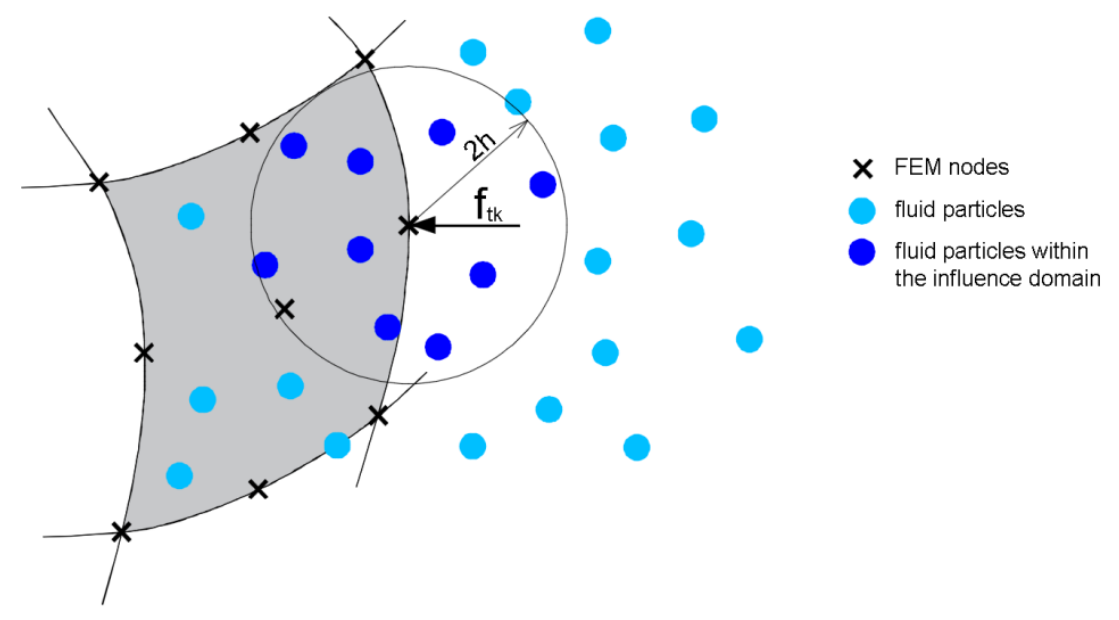

Fig. 4 Fluid forces on the structure 
The nodal forces $f_{f s}$, calculated according to expression (26), represent part of the effective load vector on the structure, whose unknown displacements are calculated by a numerical model based on the finite element method.

The interaction matrix $Q$ in equation (26) includes only the surface integration and is defined as (Figure 6):

$$
\mathbf{Q}=\int_{\Gamma_{i}} \boldsymbol{N}^{T} \vec{n} \boldsymbol{N} d \Gamma_{i}
$$

where $N$ is the matrix of global shape functions for the structure $[9,10,24]$. After determining the displacement of the structure, the calculation domain of the fluid is reconfigured.

For the particles close to the reconfigured boundary it is necessary to re-determine the density and the pressure. This procedure is effective only for small incremental displacements of the structure, so the time step of the analysis should be set so that the displacements are really small (less than one tenth of the initial fluid particle spacing).

As already mentioned, the domain of the fluid is simulated by a set of boundary particles $[3,8]$. In this paper, on the interface between structure and fluid the so-called virtual particles in multiple layers are placed (dynamic boundary conditions). In this case, the first layer of particles coincides with the inner surface of the shell structure (Figure 5).

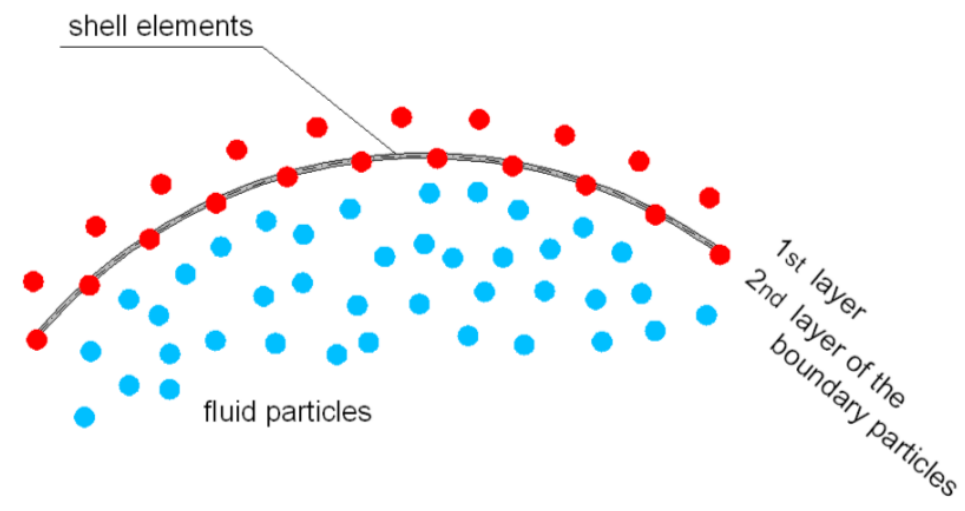

Fig. 5 Virtual fluid particles on the interface

After the calculation of the nodal displacements of the structure, the boundary particles on the interface change their position according to the calculated displacements of the structure (Figure 5). In the sense of the kinematic boundary condition, this approach completely fulfilled the condition of compatibility of the displacements on the interface. Dynamic boundary condition is not fulfilled completely (velocities), but using very small time step this error can be neglected.

By adjusting the coordinates of the boundary particles on the interaction interface, the boundary conditions for the fluid are changed (the fluid domain is changed). The next step is the fluid analysis and the calculation of the pressures with the SPH model. This procedure is repeated for each time step of the analysis, up to the total time of the calculation. The solution scheme is presented in Figure7. 


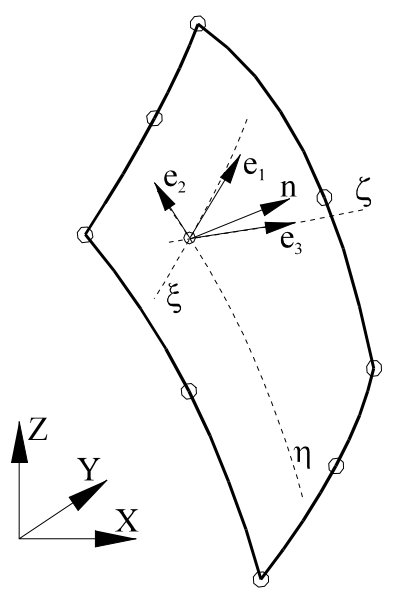

Fig. 6 The fluid-structure interaction surface and unit norm

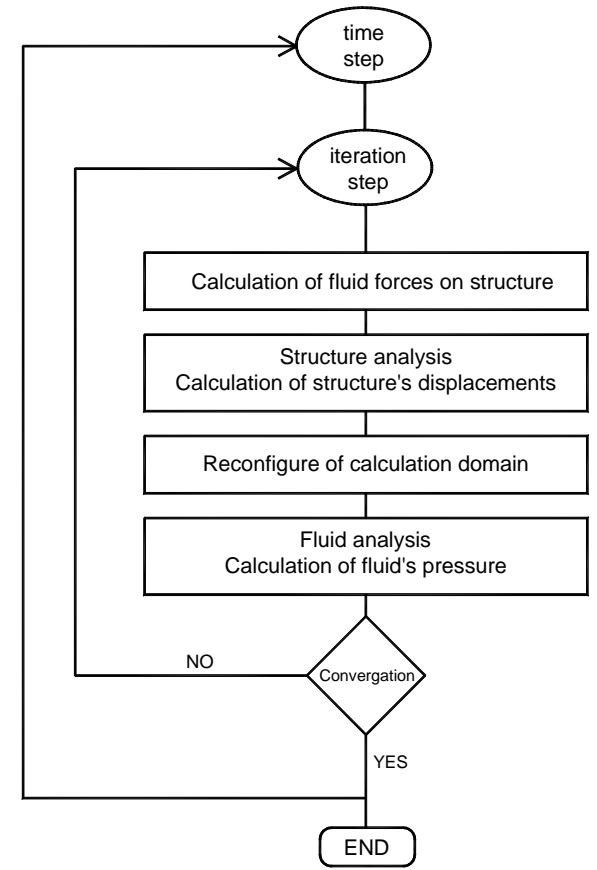

Fig. 7 The solution scheme for the fluid-structure coupled problems analysis

\section{NUMERICAL TESTS}

\subsection{INTRODUCTION}

Using the previously illustrated numerical model for interaction between the structure and the fluid, an analysis of water sloshing in tanks with rigid and deformable walls was performed according to examples from literature [30, 31, 32]. These examples can describe most of the possibilities of the developed numerical model for interaction. Sloshing in the rigid tank was analysed first and then sloshing in the deformable tank.

The tanks were exposed to harmonic accelerations of the base defined by:

$$
a=a_{0} \cdot \sin \left(\omega_{f} t\right)
$$

where $a_{0}$ is the excitation amplitude (in all numerical tests $0.05 g$ ), and $\omega_{f}$ is the excitation frequency. In all numerical tests, initial water density $\rho_{0}=1000 \mathrm{~kg} / \mathrm{m}^{3}$ and kinematic viscosity $v_{0}=10^{-6} \mathrm{~m}^{2} / \mathrm{s}$ were adopted.

\subsection{SLOSHING IN A TANK WITH RIGID WALLS}

The dimensions of the tank were: $0.8 \mathrm{~m} \times 0.5 \mathrm{~m} \times 0.7 \mathrm{~m}$, with water level of $0.3 \mathrm{~m}$ (Figure 8). The tank was exposed to the harmonic acceleration of the base according to expression (29). Initial particle spacing was $0.025 \mathrm{~m}$, arranged in a Cartesian grid (Figure 9). The total number of particles, including boundary (virtual) particles, was 15001. Adopted smoothing length $h$ is $0.0375 \mathrm{~m}$ 


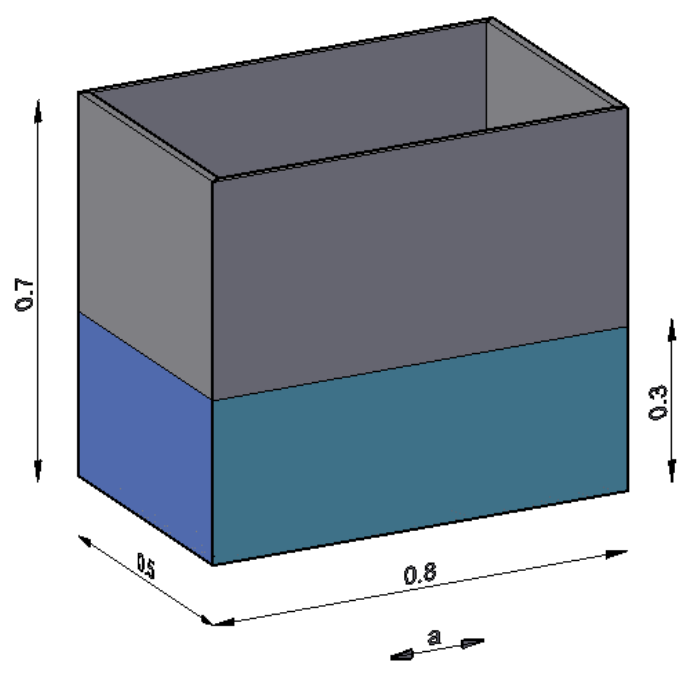

Fig. 8 Tank dimensions

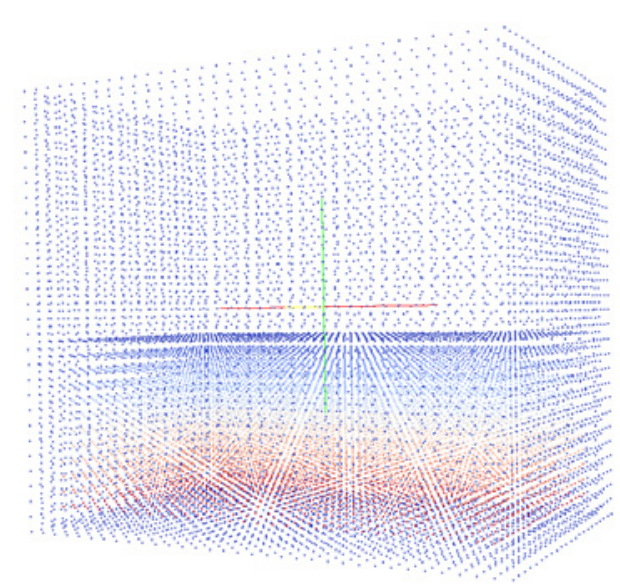

Fig. 9 Initial particle distribution

The theoretical natural frequency of the first mode of water oscillation in this tank is $\omega_{1}=0.89$ $\mathrm{Hz}$ [18]. Using the developed numeric model, water behaviour in the tank was analysed at a frequency which corresponded to the natural frequency of the tank $\left(\omega_{f}=\omega_{1}\right)$, but also with the excitation whose frequencies are very close to the natural frequency of the tank (Figure 10).

In Figure $10 A_{\max }$ is the highest water level on the right side of the tank compared to the initial water level, $w$ is the tank length $(0.8 \mathrm{~m})$. The results of the analysis are compared with the results of the 2D numerical model and the experimental results available from literature [32].

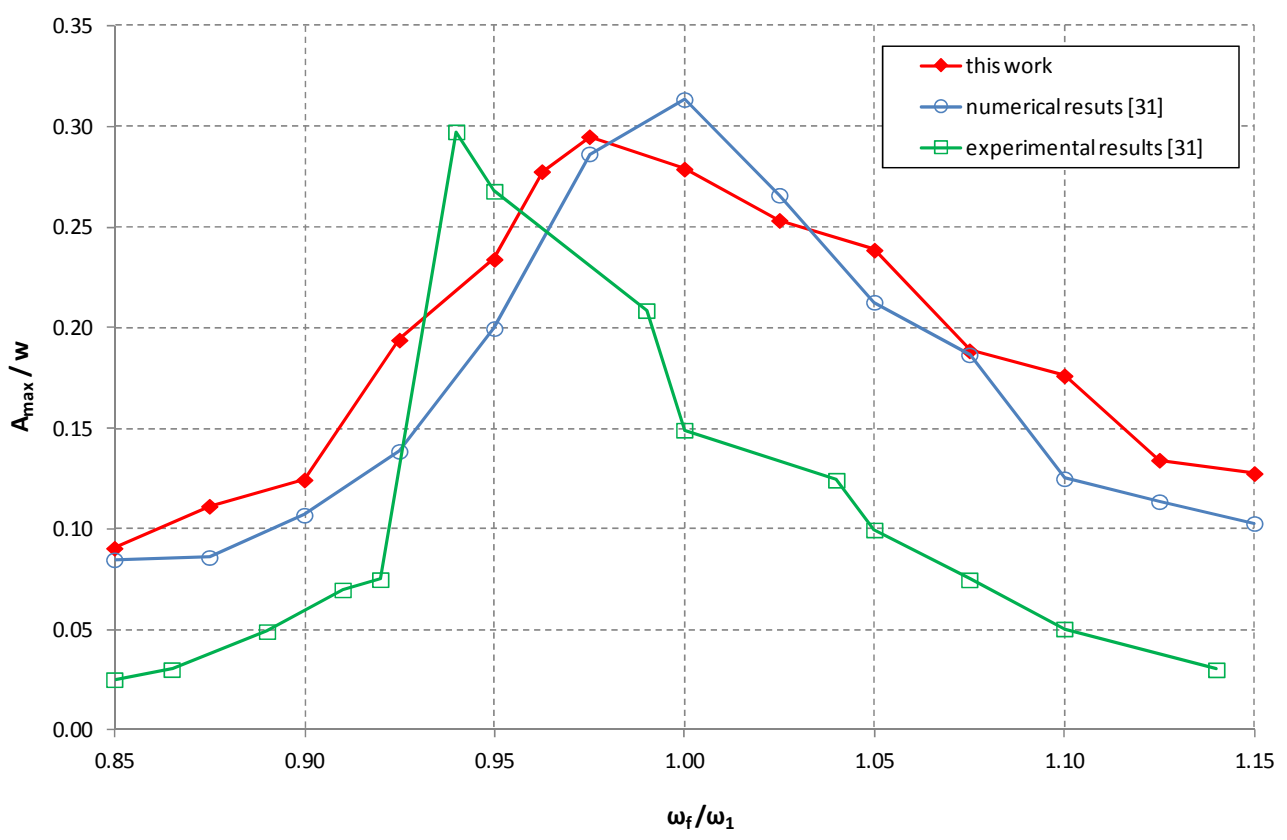

Fig. 10 Resonant response of the tank with rigid walls

From Figure 10 it is apparent that the value of the maximum water rise is $A_{\max }=0.24 \mathrm{~m}$ and that this value coincides with the experimental results from [32]. The maximum elevation of the water in the tank was obtained for a frequency slightly lower than the theoretical resonant 
frequency $\left(\omega_{f}=0.975 \omega_{1}\right)$. Thus, according to the experimental results, the resonant frequency for the observed tank is slightly lower than the theoretical resonant frequency.

From the results it is apparent that this numerical model can realistically simulate the behaviour of water in a rigid tank during a dynamic excitation.

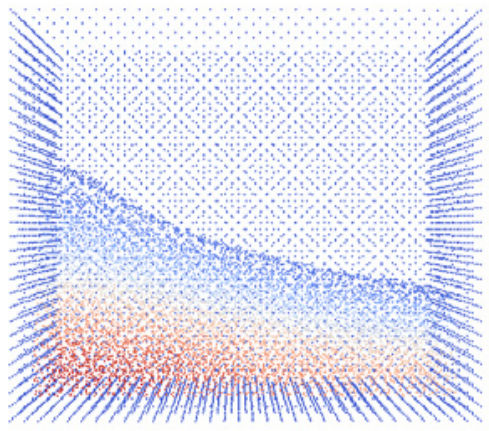

(a)

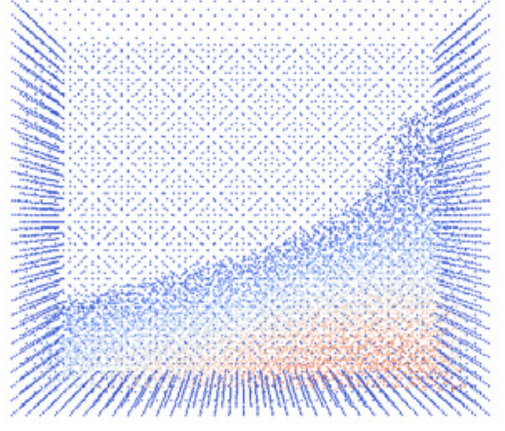

(b)

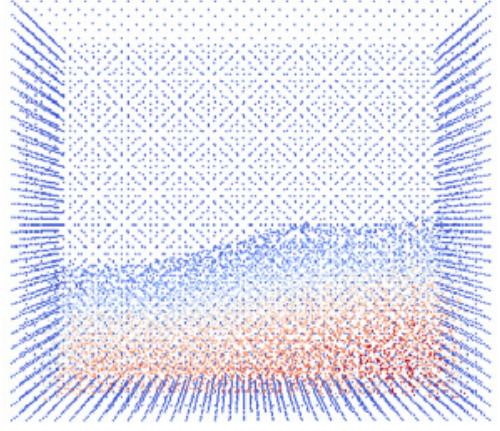

(c)

Fig. 11 Maximum elevation of water in the tank for $\omega_{f}=0.975 \omega_{1}$

Figure $11 \mathrm{~b}$ shows the tank at the time when the maximum water level was reached on the right wall of the tank, and Figures 11a and 11c just before and after that time.

\subsection{SLOSHING IN A TANK WITH DEFORMABLE WALLS}

In this example the dimensions of the tank were: $5.0 \mathrm{~m} \times 5.5 \mathrm{~m} \times 8.0 \mathrm{~m}$, with water level of 4.0 $\mathrm{m}$ (Figure 12), and it was exposed to the harmonic acceleration of the base according to expression (29). The initial particle spacing was $0.2 \mathrm{~m}$ arranged in a Cartesian grid (Figure 13). The total number of particles, including boundary (virtual) particles, was 18230. Adopted smoothing length $h$ is $0.3 \mathrm{~m}$. Deformable walls have properties showed in Table 1 and each wall is modelled with 160 finite shell elements (Figure 14).

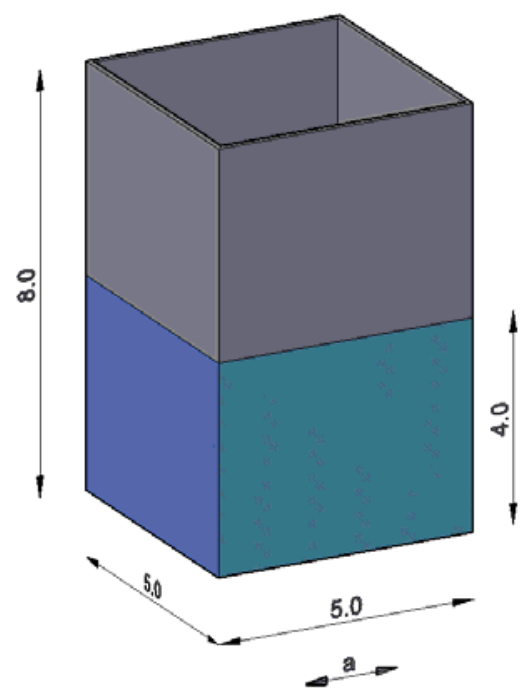

Fig. 12 Tank dimensions

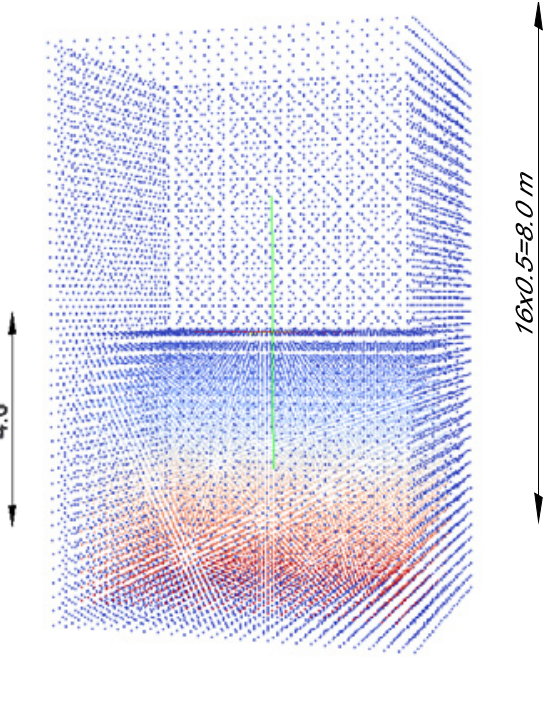

Fig. 13 Initial particle distribution

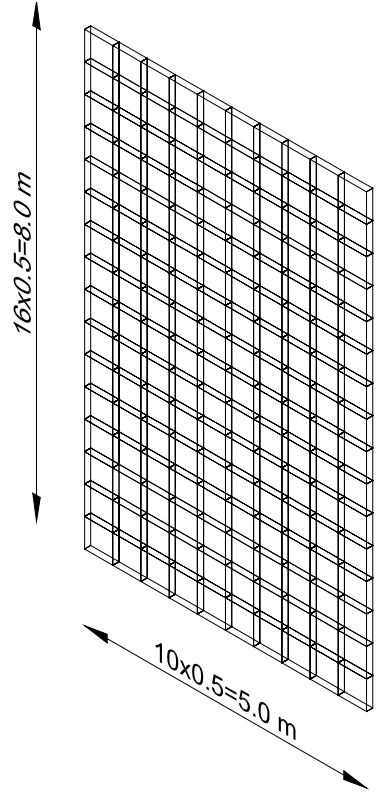

Fig. 14 FEM discretization of deformable walls 
Table 1 Material properties of deformable tank walls [32]

\begin{tabular}{cccc}
\hline Modulus of elasticity [GPa] & Poisson's coefficient & Density $\left[\mathrm{kg} / \mathrm{m}^{3}\right]$ & Thicknesses [m] \\
\hline \hline 5.0 & 0.3 & 7860 & 0.11034 \\
\hline
\end{tabular}

In the first numerical test it was adopted that all of the walls of the tank were rigid. In the second numerical test it was adopted that the two opposite walls (which were perpendicular to the direction of the excitation) were deformable shells, with the material characteristics shown in Table 1, while the other walls were rigid.

The theoretical natural frequency of the first mode of water oscillation in this tank is $\omega_{1}=0.39$ $\mathrm{Hz}$ [18]. Using the developed numeric model, water behaviour in the tank was analysed at a frequency which corresponded to the natural frequency of the tank $\left(\omega_{f}=\omega_{1}\right)$, but also with the excitation whose frequencies are very close to the natural frequency of the tank (Figure 15).

In Figure $14, A_{\max }$ is the highest water level on the right side of the tank in comparison to the initial water level and $\mathrm{w}$ is the tank length $(5.0 \mathrm{~m})$. The results of the analysis are compared with the results of the numerical model and the experimental results available from literature [32], and they show very good agreement.

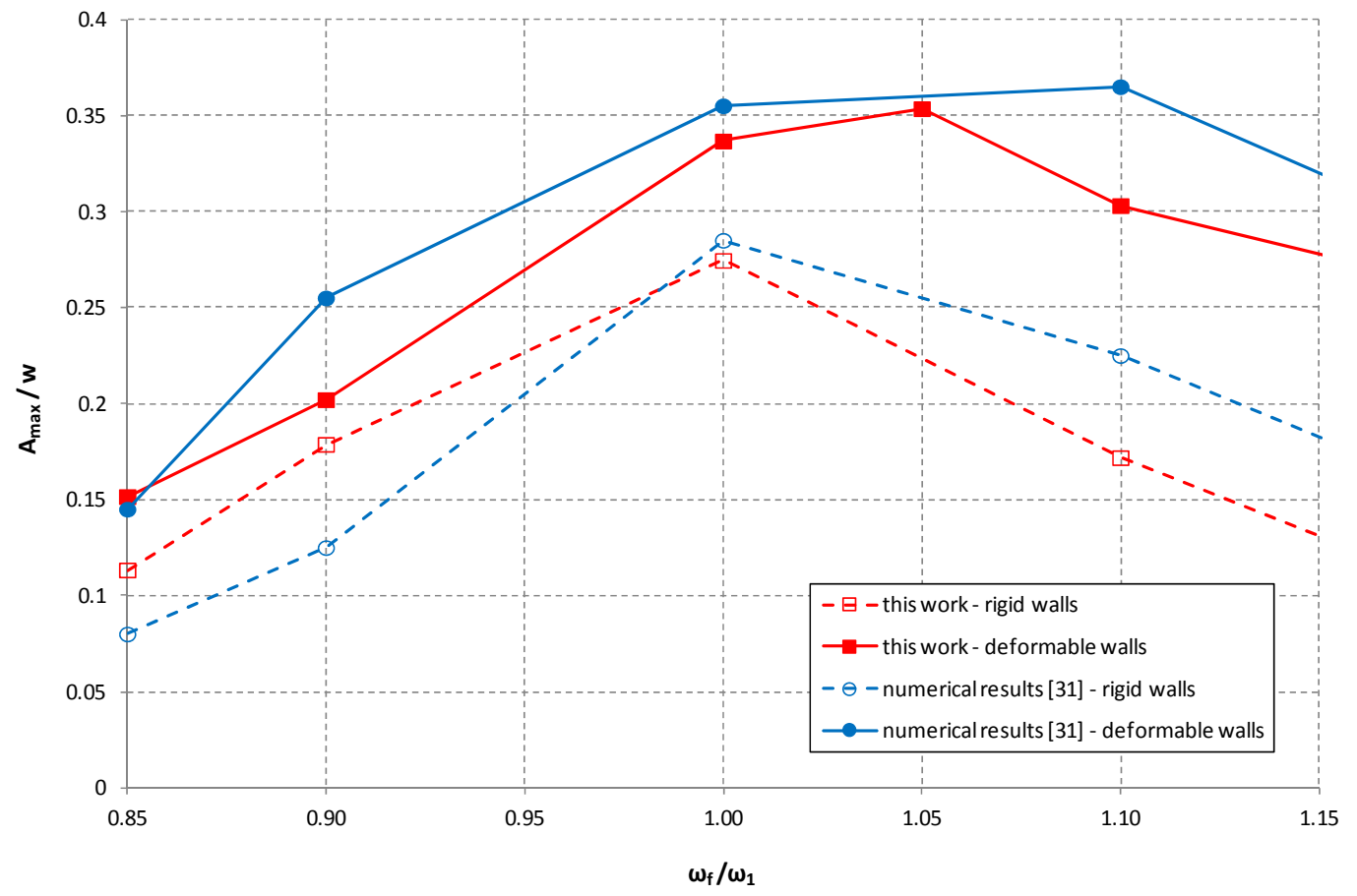

Fig. 15 Resonant response of the tank with deformable walls

The horizontal displacements of the top of the deformable structure walls over time are shown in Figure 16. It can be seen that the displacements oscillate around the value for the initial hydrostatic load displacement and are relatively regular. This can be explained by a relatively shallow water level in the original example. 


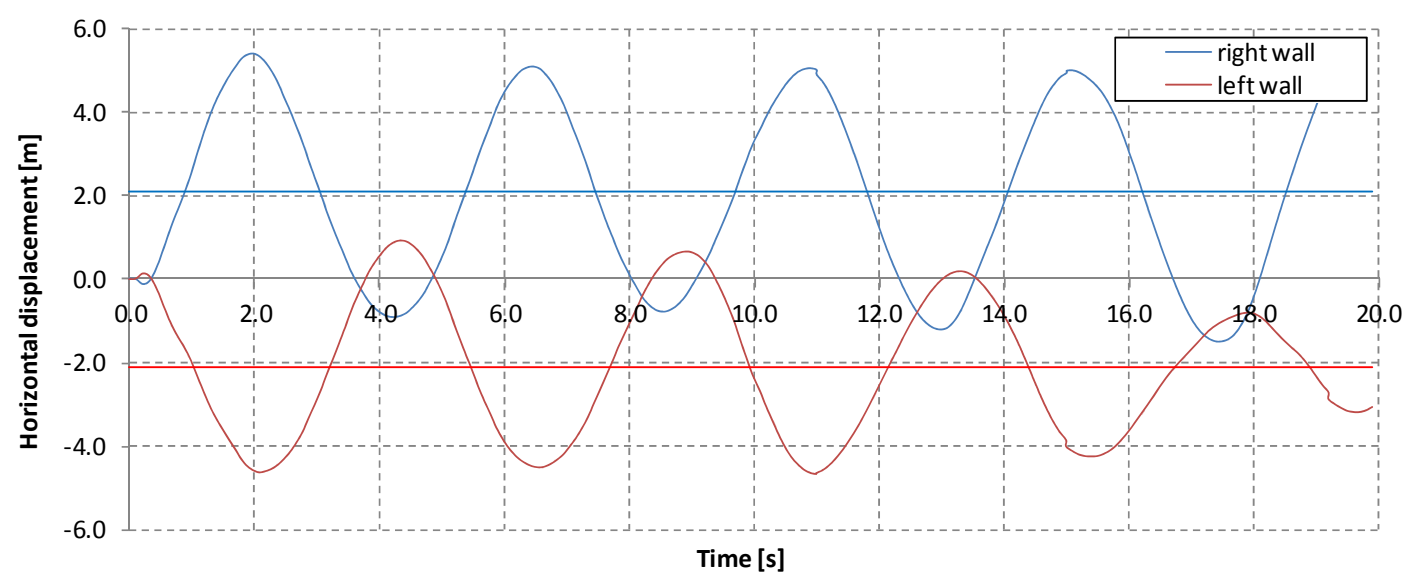

Fig. 16 The displacements of the top of the deformable walls over time

Figure 17 shows the rigid tank at the time when the maximum water level was reached on the right wall of the tank (Figure 17b), and Figures 17a and 17c just before and just after that time. Figures 18 show the water levels at the same times as Figures 17, but for the tank with deformable walls. It's obvious that the responses of the rigid tank and the deformable tank are quite different, and that the deformability of the walls of the tank has to be included in the analysis of tank behaviour.

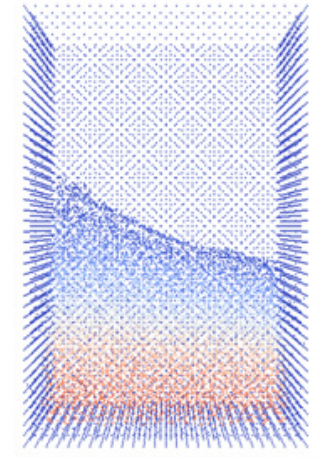

(a)

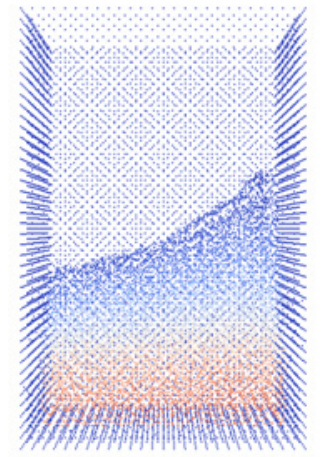

(b)

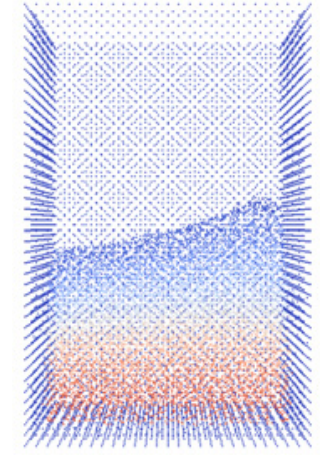

(c)

Fig. 17 Resonant response of the tank with rigid walls

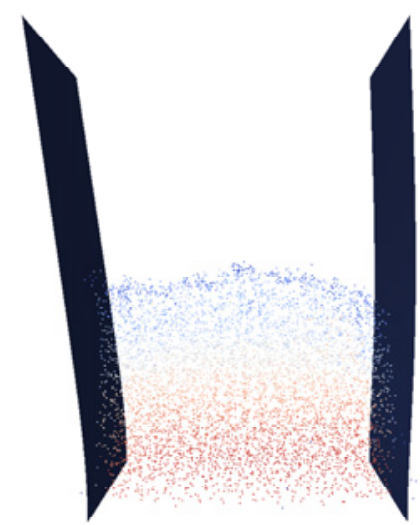

(a)

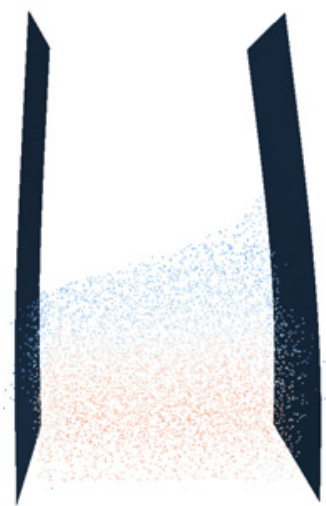

(b)

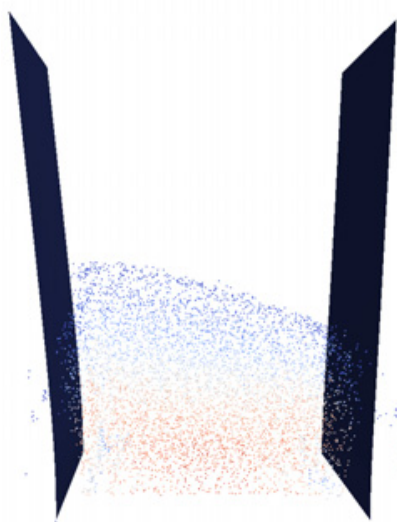

(c)

Fig. 18 Resonant response of the tank with deformable walls 


\section{CONCLUSION}

As it was emphasized in the first section, civil engineering structures that are in direct contact with fluid (liquid), such as dams, water tanks, off shore structures, pipelines, water towers, etc., are very common in engineering practice. Numerical models for a realistic simulation of these structures have to include the fluid-structure interaction for this complex system to be realistically modelled.

The numerical model presented in this paper is primarily intended for the computer simulation of the behaviour of structures that are in contact with fluid under seismic (dynamic) loads. The final model is combination of two well-known numerical methods (FEM \& SPH) and it has been used to solving practical problems related to liquid tank dynamics during earthquakes. A partitioned coupling of two previously developed models is adopted. The model based on the SPH is used for analysis of the fluid and the model based on the FEM is used for analysis of the structure. The model can simulate main nonlinear characteristics of the structure: flow (yielding) under compression and tension, and increase in plasticity. It is also possible to simulate main characteristics of the fluid (compressibility, viscous flow and turbulence). Apart from the ability to analyse the FSI problems, it also provides a simple method for analysing individual fields. One very illustrative example is given in paper [35].

Based on the results of the performed numerical tests and their comparisons with the example from literature [32], it can be concluded that the developed model presents reliable tool for simulating water flow in tank with rigid or deformable walls during resonant or non- resonant dynamic excitation.

\section{REFERENCES}

[1] G. Hou, J. Wang, A. Layton, Numerical Methods for Fluid-Structure Interaction - A Review, Commun. Comput. Phys., Vol. 12, No. 2, pp. 37-377, 2012.

https://doi.org/10.4208/cicp.291210.290411s

[2] K.J. Bathe, W.F. Hahn, On transient analysis of fluid-structure system, Computers and Structures, Vol. 10, pp. 383-391, 1979. https://doi.org/10.1016/0045-7949(79)90109-3

[3] G.R. Liu, M.B. Liu, Smoothed Particle Hydrodynamics: A Meshfree Particle Method. World Scientific, 2003. https://doi.org/10.1142/9789812564405

[4] J.C. Jo, Fluid-Structure Interaction, Pressure Vessels and Piping Systems, chapter: FluidStructure Interaction, Ed. Kwon Y.W. \& Lam, P.S., UNESCO-EOLSS, 2009.

[5] J. Degroote, R. Haelterman, S. Annerel, P. Bruggeman, J. Vierendeels, Performance of partitioned procedures in fluid-structure interaction, Computers and Structures, Vol. 88, pp. 446-457, 2010. https://doi.org/10.1016/i.compstruc.2009.12.006

[6] J. Degroote, K.J. Bathe, J. Vierendeels, Performance of a new partitioned procedure versus a monolithic procedure in fluid-structure interaction, Computers and Structures, Vol. 87, pp. 793-801, 2009. https://doi.org/10.1016/i.compstruc.2008.11.013

[7] A. Colagrossi, M. Landrini, Numerical simulation of interfacial flows by smoothed particle hydrodynamics, Journal of Computational Physics, Vol. 191, pp. 448-475, 2003.

https://doi.org/10.1016/S0021-9991(03)00324-3 
[8] A.J.C. Crespo, M. Gómez-Gesteira, R.A. Dalrymple, Boundary Conditions Generated by Dynamic Particles in SPH Methods, Tech Science Press., Vol. 5, No. 3, pp. 173-184, 2007.

[9] H-C. Huang, Static and Dynamic Analysis of Plates and Shells, Springer-Verlag, 1989. https://doi.org/10.1007/978-1-4471-1669-1

[10] J. Radnić, A. Harapin, D. Matešan, Model for Static Analyses of Concrete Shells, Engineering Modelling, Vol. 14, No. 3-4, pp. 93-99, 2000.

[11] J. Radnić, A. Harapin, D. Matešan, Geometrical nonlinearity model in static analysis of shells, Građevinar, Vol. 55, No. 10, pp. 583-589, 2003. (in Croatian)

[12] S.K. Chakrabarti (ed.), Numerical Models in Fluid Structure Interaction, Advances in Fluid Mechanics, WIT Press, 42, 2005. https://doi.org/10.2495/978-1-85312-837-0

[13] E.H. Dowell, K.C. Hall, Modelling of fluid-structure interaction, Annual Review of Fluid Mechanics, Vol. 33, pp. 445-490, 2001. https://doi.org/10.1146/annurev.fluid.33.1.445

[14] S.R. Idelsohn, E. Onate, F.A. Del Pin, Lagrangian meshless finite element method applied to fluid-structure interaction problems, Computers and Structures, Vol. 81, pp. 655-671, 2003. https://doi.org/10.1016/S0045-7949(02)00477-7

[15] J.H. Ferziger, M. Perić, Computational Methods for Fluid Dynamics, Springer-Verlag, 2002. https://doi.org/10.1007/978-3-642-56026-2

[16] J. Braithwaite, An Introduction to Hydrodynamics, Online: http://elibrary.bsu.az/books 400/N_224.pdf, 2011.

[17] K.J. Bathe, H. Zhang, M.H. Wang, Finite element analysis of incompressible and compressible fluid flows with free surfaces and structural interactions, Comput. Struct., Vol. 56, No. 2-3, pp. 193-213, 1995. https://doi.org/10.1016/0045-7949(95)00015-9

[18] R.A. Ibrahim, Liquid Sloshing Dynamics: Theory and Applications, Cambridge University Press, 2000.

[19] L. Lucy, A numerical approach to testing of the fusion process, Journal Astronomical., Vol. 88, pp. 1013-1924, 1977. https://doi.org/10.1086/112164

[20] R.A. Gingold, J.J. Monaghan, Smoothed particle hydrodynamics, theory and application to non-spherical stars, Mon Nat R Astr Soc, Vol. 181, pp. 375-389, 1997.

https://doi.org/10.1093/mnras/181.3.375

[21] E. Onate, S.R. Idelsohn, F. Del Pin, R. Aubry, The particle finite element method. An overview, Int J Comput Methods, Vol. 1, No. 2, pp. 267-307, 2004.

https://doi.org/10.1142/S0219876204000204

[22] A.J.C. Crespo, Application of the Smoothed Particle Hydrodynamics model SPHysics to free-surface hydrodynamics, pHD thesis, Universidade De Vigo, Departamento De Fisica Aplicada, 2008.

[23] J.J. Monaghan, On the problem of penetration in particle methods, Journal of Computational Physics, Vol. 82, pp. 1-15, 1989. https://doi.org/10.1016/0021-9991(89)90032-6

[24] K.J. Bathe, E.N. Dvorkin, A formulation of general shell elements - The use of mixed interpolation of tensorial components, Int J Numer Meth Eng, Vol. 22, pp. 697-722, 1986. https://doi.org/10.1002/nme.1620220312 
[25] B. Brank, J. Korelc, A. Ibrahimbegović, Dynamics and time-stepping schemes for elastic shells undergoing finite rotations, Computers and Structures, Vol. 81, pp. 1193-1210, 2003. https://doi.org/10.1016/S0045-7949(03)00036-1

[26] J.C. Nagtegaal, J.E. De Jong, Some Computational Aspects of Elasto-plastic Large Strain Analysis, International Journal for Numerical Methods in Engineering, Vol. 17, pp. 15-41, 1981. https://doi.org/10.1002/nme.1620170103

[27] S. Valiapan, P. Boonlaulohr, I.K. Lee, Nonlinear analysis for anisotropic materials, Int J Numer Meth Engng, Vo. 10, pp. 597-606, 1989.

https://doi.org/10.1002/nme.1620100309

[28] R. Hill, The mathematical theory of plasticity, Clarendon Press, Oxford. 1950

[29] J. Lubliner, Plasticity Theory, Courier Dover Publications, 2008

[30] S. Koshizuka, Y. Oka, Moving particle semi-implicit method for fragmentation of incompressible fluid, Nucl Sci Eng, Vol. 123, pp. 421-434, 1996.

https://doi.org/10.13182/NSE96-A24205

[31] S. Koshizuka, A. Nobe, Y. Oka, Numerical analysis of breaking waves using the moving particle semi-implicit method, Int J Numer Meth Fluids, Vol. 26, pp. 751-769, 1998.

https://doi.org/10.1002/(SICI)1097-0363(19980415)26:7<751::AIDFLD671>3.0.CO;2-C

[32] C.J.K. Lee, H. Noguchi, S. Koshizuka, Fluid-shell structure interaction analysis by coupled particle and finite element method, Comput Struct, Vol. 85, pp. 688-697, 2007.

https://doi.org/10.1016/j.compstruc.2007.01.019

[33] A.J.C. Crespo, J.M. Domínguez, B.D. Rogers, M. Gómez-Gesteira, S. Longshaw, R. Canelas, R. Vacondio, A. Barreiro, O. García-Feal, DualSPHysics: open-source parallel CFD solver on Smoothed Particle Hydrodynamics (SPH), Computer Physics Communications, Vol. 187, pp. 204-216, 2015. https://doi.org/10.1016/i.cpc.2014.10.004

[34] J.M. Domínguez, A.J.C. Crespo, D. Valdez-Balderas, B.D. Rogers and M. Gómez-Gesteira, New multi-GPU implementation for Smoothed Particle Hydrodynamics on heterogeneous clusters, Computer Physics Communications, Vol. 184, pp. 1848-1860, 2013. https://doi.org/10.1016/j.cpc.2013.03.008

[35] M. Sunara Kusić, J. Radnić, N. Grgić, A. Harapin, Sloshing in medium size tanks caused by earthquake studied by SPH, Građevinar, Vol. 70, No. 8, 2018.

https://doi.org/10.14256/JCE.2169.2017 